\title{
Supersymmetry breaking induced by radiative corrections
}

\author{
Borut Bajc, $^{a, b}$ Stéphane Lavignac ${ }^{c}$ and Timon Mede ${ }^{a}$ \\ ${ }^{a}$ Jožef Stefan Institute, \\ 1000 Ljubljana, Slovenia \\ ${ }^{b}$ Department of Physics, University of Ljubljana, \\ 1000 Ljubljana, Slovenia \\ ${ }^{c}$ Institut de Physique Théorique, ${ }^{1}$ CEA-Saclay, \\ F-91191 Gif-sur-Yvette Cedex, France \\ E-mail: borut.bajc@ijs.si, stephane.lavignac@cea.fr, timon.mede@ijs.si
}

\begin{abstract}
We show that simultaneous gauge and supersymmetry breaking can be induced by radiative corrections, à la Coleman-Weinberg. When a certain correlation among the superpotential parameters is present, a local supersymmetry-breaking minimum is induced in the effective potential of a gauge non-singlet field, in a region where the tree-level potential is almost flat. Supersymmetry breaking is then transmitted to the MSSM through gauge and chiral messenger loops, thus avoiding the suppression of gaugino masses characteristic of direct gauge mediation models. The use of a single field ensures that no dangerous tachyonic scalar masses are generated at the one-loop level. We illustrate this mechanism with an explicit example based on an SU(5) model with a single adjoint. An interesting feature of the scenario is that the GUT scale is increased with respect to standard unification, thus allowing for a larger colour Higgs triplet mass, as preferred by the experimental lower bound on the proton lifetime.
\end{abstract}

KEYWORDS: Supersymmetry Breaking, GUT

ARXIV EPRINT: 1202.2845

\footnotetext{
${ }^{1}$ Laboratoire de la Direction des Sciences de la Matière du Commissariat à l'Energie Atomique et Unité de Recherche associée au CNRS (URA 2306).
} 


\section{Contents}

1 Introduction 1

2 The method 2

$3 \quad$ An SU(5) example with a single adjoint 3

4 Intermediate states, the GUT scale and proton decay 5

5 Model-building aspects $\quad 8$

5.1 Fermion masses 8

$\begin{array}{ll}5.2 \text { Doublet-triplet splitting } & 10\end{array}$

$\begin{array}{lll}6 & \text { The superpartner spectrum } & 11\end{array}$

$\begin{array}{lll}7 & \text { Conclusions } & 13\end{array}$

\section{Introduction}

Supersymmetric Grand Unification, although appealing, is plagued by large uncertainties associated with the necessity of breaking supersymmetry. It is an old idea [1] to try and unify the supersymmetry and the gauge symmetry breaking sectors, thus minimizing and optimizing them. To our knowledge, apart from ref. [2], all attempts so far were using gauge singlets (see for example refs. $[1,3-7]$ ) and/or were following nonperturbative approaches (see for example refs. [8-12]). In this paper we would like to investigate further the possibility of breaking simultaneously global supersymmetry and the gauge symmetry in the framework of perturbative gauge theories without gauge singlets.

There are several good reasons for using the same field(s) to spontaneously break supersymmetry and the gauge symmetry. The first obvious motivation is economy: there is no need for two separate sectors. The second reason is that this automatically provides a mechanism for mediating supersymmetry breaking to the observable sector, with the heavy gauge fields playing the role of messenger fields. The MSSM soft terms can then be predicted in terms of a small number of parameters (up to supergravity corrections), and as a bonus the gaugino mass problem of gauge mediation with chiral messenger fields ${ }^{1}$ is absent. Finally, another motivation is that the messenger mass scale is identified with the unification scale, thus helping to avoid a possible stability problem of the Minkowski vacuum in which supersymmetry is broken $[16,17]$.

\footnotetext{
${ }^{1}$ Namely the fact, already noticed in the early literature, that gaugino masses vanish at leading order in direct gauge mediation models with tree-level supersymmetry breaking [13]. Radiative corrections may however solve this problem [14, 15].
} 
If one sticks to perturbative approaches, several identical representations (e.g. adjoints $^{2}$ ) of the unified gauge group are required if supersymmetry is broken at the tree level, as is usually assumed. The minimal model of this kind is the SU(5) Grand Unified example with two adjoints given in ref. [2]. The presence of several adjoints, however, generally implies tachyonic sfermion masses at the one-loop level [18]. Since gaugino masses arise at the same order of perturbation theory, this problem cannot be cured by the renormalization group running between the messenger scale and low energy. While there are various ways to make this negative contribution subdominant compared with other twoloop contributions (for example by imposing a mild hierarchy between the vevs of the two fields), it is difficult to build a consistent model.

In this paper, we avoid this problem by considering the possibility that supersymmetry and the gauge symmetry are simultaneously broken by radiative corrections, à la ColemanWeinberg [19]. This makes it possible to use a single representation for this purpose, thus avoiding large negative one-loop gauge messenger contributions to soft scalar masses. ${ }^{3}$ Furthermore, we do not make use of gauge singlet fields.

The plan of the paper is the following. In section 2, we describe the general method for finding gauge and supersymmetry breaking minima in the one-loop effective potential of a single field. This is then applied to the case of the adjoint representation of SU(5) in section 3, where the conditions for the mechanism to work are identified. In the next sections, we discuss several consequences of implementing this mechanism in an SU(5) unified theory, taking as an example the minimal model (to be considered as an existence proof) in which it can work, at a price of tuning the superpotential parameters. We first show in section 4 that intermediate-mass states are unavoidably present, which affects the running of the Standard Model gauge couplings and raises the GUT scale, thus allowing to increase the mass of the colour Higgs triplet that mediates proton decay. We then comment on the fermion mass relations and on the doublet-triplet splitting in section 5. Finally, we discuss the superpartner spectrum in section 6 and give our conclusions in section 7 .

\section{The method}

The method we will use to seek local supersymmetry breaking minima in the one-loop effective potential can be seen as a generalized version of Witten's mechanism for generating the hierarchy between the weak scale and the GUT scale [1]. Witten's method consists in finding a flat direction that breaks both supersymmetry and the gauge group, and in stabilizing it by radiative corrections. At the one-loop level the effective potential has the following approximate form:

$$
V(\sigma)=\frac{|F|^{2}}{1+\left(c_{g}-c_{\lambda}\right) \log \left(|\sigma|^{2} / \mu^{2}\right)},
$$

\footnotetext{
${ }^{2} \mathrm{~A}$ single adjoint is not enough to break both supersymmetry and the unified gauge symmetry at the tree level, even in the presence of gauge singlets.

${ }^{3}$ Note that in the case of $\mathrm{SO}(10)$ gauge symmetry one usually needs more than one representation to spontaneously break the gauge group, so that negative one-loop scalar masses are generated and must be suppressed.
} 
where $c_{x}=c_{x}^{0} x^{2} / 16 \pi^{2}(x=g$ or $\lambda)$ are loop-suppressed factors, $\sigma$ is the flat direction, and $\mu$ is the renormalization scale. For the mechanism to work, it is crucial that both $g$ and $\lambda$ participate. Typically, the gauge coupling goes at high energy towards asymptotic freedom while the Yukawa coupling behaves the opposite way, so that $c_{g}-c_{\lambda}$ changes sign and becomes negative above some scale, thus stabilizing the value of $\sigma$ (which otherwise would be pushed to higher values by $\left.c_{g}-c_{\lambda}>0\right)$. The minimum is reached at $|\sigma|=\mu_{0}$ where

$$
c_{g}\left(\mu_{0}\right)=c_{\lambda}\left(\mu_{0}\right) .
$$

Notice that $F$ in eq. (2.1) is a constant ( $\sigma$ is a tree-level flat direction). Witten's mechanism cannot work if there is no flat direction (this is the typical situation in the minimal case of a single field ${ }^{4}$ ), or when for some reason the minimum (2.2) cannot be reached.

The method we are going to use in this paper relies on the observation that a minimum at a finite value of $\sigma$ can also appear in the case where $F$ is a slowly varying function of $\sigma$. As we will see, this can work even for negligible Yukawa couplings, i.e. for an effective potential of the form:

$$
V(\sigma)=\frac{|F(\sigma)|^{2}}{1+c_{g} \log \left(|\sigma|^{2} / \mu^{2}\right)} .
$$

In the first approximation one could even neglect the scale dependence of $c_{g}$, i.e. of the gauge coupling, since its change is not crucial anymore. It is now the field dependence in $F(\sigma)$ that counterbalances the one-loop logarithmic contribution in the denominator. For this to work, the tree-level potential should be almost flat. In practice this is the case when the first few derivatives of the superpotential are small. We are not even close to an extremum of the tree-level potential, but this is corrected by the one-loop logarithmic contribution. In supersymmetric theories one often expands the scalar potential around tree-level flat directions, but this case is actually closer to the original Coleman-Weinberg philosophy, in which one expands the potential in a region where the first tree-level derivatives are non-vanishing.

\section{An $\mathrm{SU}(5)$ example with a single adjoint}

Let us illustrate the method exposed in the previous section with an $\mathrm{SU}(5)$ example. The chiral superfield $\sigma$ is identified with the Standard Model singlet direction of an adjoint $\Sigma$ :

$$
\Sigma=\frac{\sigma}{\sqrt{30}} \operatorname{diag}(2,2,2,-3,-3)+\cdots .
$$

In the small $\left|F(\sigma) / \sigma^{2}\right|$ limit, which is the phenomenologically relevant one ${ }^{5}$ the one-loop effective potential for $\sigma$ is given to a very good approximation by:

$$
V(\sigma)=\frac{|F(\sigma)|^{2}}{1+c \log \left(|\sigma|^{2} / \mu^{2}\right)},
$$

\footnotetext{
${ }^{4}$ An exception to this rule is the cubic superpotential of the adjoint of $\mathrm{SU}(6)$.

${ }^{5}$ Since the messenger fields are the components of the heavy gauge supermultiplets, their mass scale is $v \equiv\langle\sigma\rangle \sim M_{\mathrm{GUT}}$ and the superpartner masses are given by $m_{\mathrm{soft}} \sim\left(g^{2} / 16 \pi^{2}\right) F(v) / v$. Hence $F(v)$ must be much smaller than $v^{2}$ if supersymmetry is realized at low energy.
} 
were $c$ only receives gauge contributions and is therefore positive. We are looking for an $\mathrm{SU}(5)$-breaking local minimum $\langle\sigma\rangle=v \neq 0$ in which supersymmetry is broken, i.e. $F(v) \neq 0$. The extremum condition reads:

$$
F^{\prime}(v)=c F(v) / v
$$

where the renormalization scale has been chosen to be $\mu=v$, while the requirement that the extremum is a minimum tells us that

$$
\left|\frac{c^{2}}{v^{2}} F(v)\right| \geq\left|F^{\prime \prime}+\frac{c}{v^{2}} F(v)\right|
$$

The general solution to this constraint can be parametrized as:

$$
F^{\prime \prime}(v)=\left(a c^{2}-c\right) F(v) / v^{2}, \quad|a| \leq 1
$$

The conditions (3.3) and (3.5) require some level of correlation between the Lagrangian parameters. To make this correlation explicit, we can expand:

$$
F(\sigma)=\sum_{n=0}^{\infty} \frac{F^{(n)}(v)}{n !}(\sigma-v)^{n},
$$

and use eqs. (3.3) and (3.5) to express $F^{\prime}(v)$ and $F^{\prime \prime}(v)$ on the right-hand side in terms of $F(v)$ and $v$. Let us see this on the following example (the presence of non-renormalizable terms in the superpotential will be justified later):

$$
W=\frac{\mu}{2} \operatorname{Tr} \Sigma^{2}+\sqrt{30} \frac{\lambda}{3} \operatorname{Tr} \Sigma^{3}+\frac{\kappa_{1}}{4 M} \operatorname{Tr} \Sigma^{4}+\frac{\kappa_{2}}{4 M}\left(\operatorname{Tr} \Sigma^{2}\right)^{2} .
$$

We thus have:

$$
F(\sigma)=\mu \sigma-\lambda \sigma^{2}+\frac{\kappa}{M} \sigma^{3}
$$

where

$$
\kappa=\frac{7}{30} \kappa_{1}+\kappa_{2} .
$$

Inserting eqs. (3.3), (3.5) and (3.8) into eq. (3.6), we obtain:

$$
\begin{aligned}
\mu & =\frac{F(v)}{2 v}\left(6-5 c+a c^{2}\right), \\
\lambda & =\frac{F(v)}{v^{2}}\left(3-4 c+a c^{2}\right), \\
\frac{\kappa}{M} & =\frac{F(v)}{2 v^{3}}\left(2-3 c+a c^{2}\right) .
\end{aligned}
$$

Since $F(v) \ll v^{2}$, all superpotential parameters must be suppressed: $\mu \ll M, \lambda \ll 1$, $\kappa v / M \ll 1$. We can therefore calculate $c$ neglecting all superpotential parameters, in which case only the $X$ and $Y$ massive vector supermultiplets contribute to the effective potential, with masses $m_{X}=m_{Y}=\frac{5}{6} g_{5}^{2}|\sigma|^{2}$, where $g_{5}$ is the $\mathrm{SU}(5)$ gauge coupling. In the approximation of small $\left|F(\sigma) / \sigma^{2}\right|$, this yields $V(\sigma)=|F(\sigma)|^{2}\left(1+\frac{12}{16 \pi^{2}} \frac{5}{6} g_{5}^{2}\left[\log \left(\frac{5}{6} g_{5}^{2}|\sigma|^{2} / \Lambda^{2}\right)+1\right]\right)^{-1}$ 


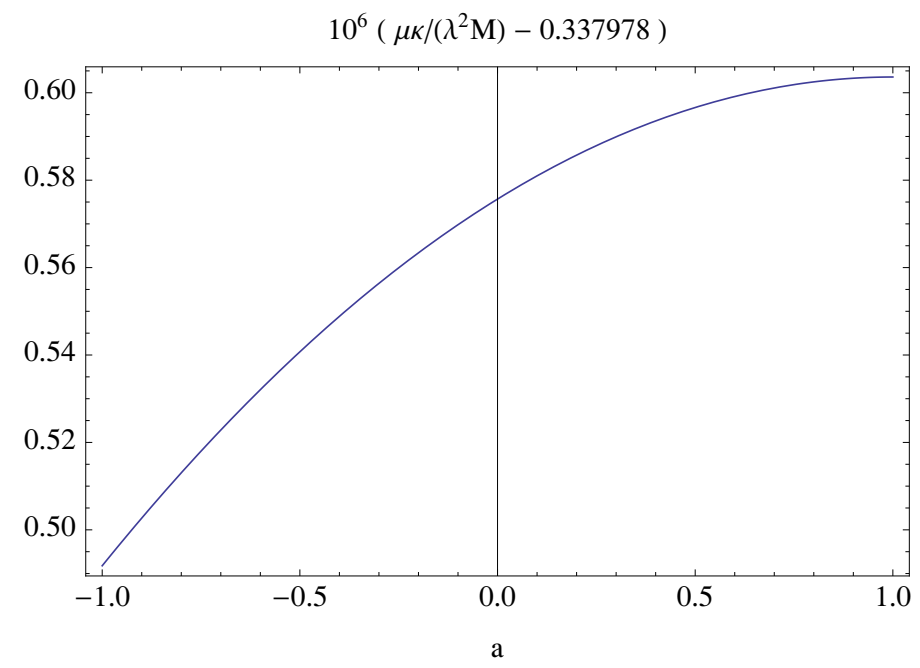

Figure 1. The allowed values of the combination $\mu \kappa /\left(\lambda^{2} M\right)$ for $c=0.04$ show the amount of finetuning needed among superpotential parameters in order to obtain a metastable supersymmetry breaking minimum with a single adjoint of $\mathrm{SU}(5)$.

(where 12 is the multiplicity of the $X$ and $Y$ gauge fields), which to a very good approximation leads to eq. (3.2) with

$$
c=\frac{12}{16 \pi^{2}} \frac{5}{6} g_{5}^{2}=10 \frac{\alpha_{\mathrm{GUT}}}{4 \pi} \approx 0.04,
$$

where $\alpha_{\mathrm{GUT}}=g_{5}^{2} /(4 \pi)$. The correlation between $\mu, \lambda$ and $\kappa / M$ arises from the fact that $c$ is a small loop-suppressed parameter, and that $a$ is bounded to have a modulus less or equal to $1 .^{6}$ This is shown in figure 1 , where the combination $\mu \kappa /\left(\lambda^{2} M\right)$ is plotted as a function of $a$ for $c=0.04$. The corresponding fine-tuning can be estimated by $d \log \left(\mu \kappa /\left(\lambda^{2} M\right)\right) /\left.d a\right|_{a=0} \simeq c^{4} / 18=\mathcal{O}\left(10^{-7}\right)$.

figure 2 shows the appearance of a local supersymmetry breaking minimum in the oneloop effective potential when conditions (3.3) and (3.5), or equivalently eqs. (3.10)-(3.12), are satisfied. If we restore the tree-level value $c=0$, the left figure remains visibly identical, while the right one displays a saddle point instead of a local minimum, as it should.

One may wonder whether the non-renormalizable terms in the superpotential (3.7) are really necessary for our purpose. The renormalizable case would imply $\kappa=0$, or due to eq. (3.12):

$$
a c^{2}-3 c+2=0 \quad \Rightarrow \quad c>0.56
$$

hence the model would become non-perturbative (remember that $c$ contains a loop factor). Thus perturbativity forces us to a superpotential with non-renormalizable operators.

\section{Intermediate states, the GUT scale and proton decay}

The fact that the superpotential parameters $\mu, \lambda$ and $\kappa$ must be suppressed to ensure $F(v) \ll v^{2}$ has important consequences for the mass spectrum and for gauge coupling

\footnotetext{
${ }^{6}$ This suggests that a similar correlation will also be present in other realizations of the mechanism.
} 

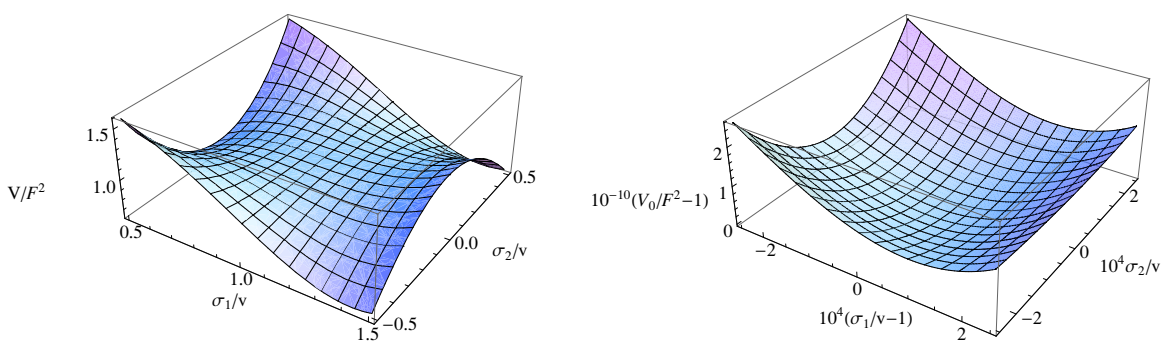

Figure 2. The one-loop effective potential for $\sigma$ in a larger (left) and smaller (right) region around the local minimum, for $a=0$ and $c=0.04 . \sigma_{1}$ and $\sigma_{2}$ are the real and imaginary parts of $\sigma$, respectively.

unification, similarly to the case of tree-level supersymmetry breaking in a renormalizable model with two adjoints [2] and in the supergravity model with a single adjoint of ref. [20]. Indeed, $\Sigma$ contains a weak triplet $\Sigma_{3}$ and a colour octet $\Sigma_{8}$ whose masses $m_{3}$ and $m_{8}$ are determined by the parameters $\mu, \lambda, \kappa_{1}$ and $\kappa_{2}$. Since, according to eqs. (3.10)-(3.11),

$$
\mu \sim \lambda v \sim \frac{F(v)}{v} \sim\left(\frac{\alpha}{4 \pi}\right)^{-1} m_{\mathrm{soft}}
$$

only $\kappa_{1}$ and $\kappa_{2}$ can give a potentially large (i.e. much larger than the superpartner mass scale $\left.m_{\text {soft }}\right)$ contribution to $m_{3}$ and $m_{8}$. Indeed, while the combination $\kappa=7 \kappa_{1} / 30+\kappa_{2}$ is constrained by eq. (3.12) to be small, $\kappa v^{2} / M \sim F(v) / v, \kappa_{1}$ and $\kappa_{2}$ could in principle be much larger. Even in the case of a strong cancellation between $\kappa_{1}$ and $\kappa_{2}$, however, $m_{3}$ and $m_{8}$ are at most of order $\kappa_{1,2} v^{2} / M \lesssim M_{\mathrm{GUT}}^{2} / M$, i.e. below the GUT scale, which affects gauge coupling unification.

More precisely, in the absence of a cancellation between $\kappa_{1}$ and $\kappa_{2}$, the colour octet and weak triplet are light with masses of order $F(v) / v$. Gauge couplings can still unify with superpartner masses in the $\mathrm{TeV}$ range, but this happens above the Planck scale. We are thus led to consider the fine-tuned case in which $\kappa_{1} \simeq-30 \kappa_{2} / 7 \gg \kappa$ in order to allow for unification below the Planck scale. The triplet and octet masses are then given by (identifying $M$ with the Planck mass $M_{P}$ ):

$$
m_{3} \simeq 4 m_{8} \simeq \frac{2}{3} \kappa_{1} \frac{v^{2}}{M}
$$

Inspection of the renormalization group equations (RGEs) shows that unification is achieved for the following values of the GUT scale and colour Higgs triplet: ${ }^{7}$ mass $m_{T}$ [21-23]

$$
\begin{aligned}
M_{\mathrm{GUT}} & =M_{\mathrm{GUT}}^{0}\left(\frac{M_{\mathrm{GUT}}^{0}}{\sqrt{m_{3} m_{8}}}\right)^{1 / 2}=M_{\mathrm{GUT}}^{0}\left(\frac{3 M_{P}}{\kappa_{1} M_{\mathrm{GUT}}^{0}}\right)^{1 / 4}, \\
m_{T} & =m_{T}^{0}\left(\frac{m_{3}}{m_{8}}\right)^{5 / 2}=32 m_{T}^{0}
\end{aligned}
$$

\footnotetext{
${ }^{7}$ We are referring here to the SU(5) partners of the MSSM Higgs doublets, which should not be confused with the weak triplet $\Sigma_{3}$ living in the adjoint representation.
} 
where $M_{\mathrm{GUT}}^{0}$ and $m_{T}^{0}$ refer to the values of the GUT scale and Higgs triplet mass in the case $m_{3}=m_{8}$. The RGE analysis of ref. [24] gives ${ }^{8} m_{T}^{0} \leq 3.6 \times 10^{15} \mathrm{GeV}$ (90\% C.L.), while $M_{\mathrm{GUT}}^{0} \approx 2 \times 10^{16} \mathrm{GeV}$. Hence the triplet mass can be increased to $10^{17} \mathrm{GeV}$, a welcome feature for proton decay. Using eq. (4.3), we can see that $M_{\mathrm{GUT}} \approx m_{T} \approx 10^{17} \mathrm{GeV}$ is obtained for $\kappa_{1} \approx 0.3$. An important feature of the model is the increase of the GUT scale. This can be understood by the unavoidable presence of non-renormalizable terms in the adjoint superpotential. They automatically lead to intermediate states which postpone unification, as can be seen from eq. (4.3).

The amount of fine-tuning between $\kappa_{1}$ and $\kappa_{2}$ that is needed in order to allow for large enough intermediate state masses, so that unification occurs at least one order of magnitude below the Planck mass, can be estimated by:

$$
\frac{\kappa}{\kappa_{1}} \sim \frac{F(v) M}{\kappa_{1} v^{3}} \lesssim\left(\frac{\alpha}{4 \pi}\right)^{-1} \frac{m_{\text {soft }} M_{P}}{\kappa_{1} M_{\mathrm{GUT}}^{2}} \sim 10^{-10}\left(\frac{m_{\mathrm{soft}}}{1 \mathrm{TeV}}\right)
$$

in which we have inserted $\kappa_{1}=0.3$ and $M_{\mathrm{GUT}}=10^{17} \mathrm{GeV}$. The inequality in eq. (4.5) takes into account the fact that soft terms receive other contributions than the gauge-mediated ones, e.g. from supergravity, and that these may dominate (see section 6). However we have seen in section 3 that the parameter $\kappa$ must be correlated with $\mu$ and $\lambda$ with a precision of $10^{-7}$ in order for a supersymmetry breaking minimum to be induced in the one-loop effective potential of $\sigma$. Hence one may view these two tunings - the one needed to ensure the existence of the supersymmetry breaking minimum and the one needed to allow for unification below the Planck scale - as a single fine-tuning of order $10^{-17}$ between $\kappa_{1}$ and $\kappa_{2}$.

The increase of the GUT scale and of the colour triplet mass has several important consequences. On the one side, a heavier Higgs triplet implies a stronger suppression of the $D=5$ proton decay operators and reduces the tension with the experimental upper bound on the proton lifetime. On the other side, $M_{\mathrm{GUT}} \sim 10^{17} \mathrm{GeV}$ means that the supergravity contribution to soft masses will dominate over the gauge-mediated ones (see the discussion in section 6), unless one relies on a mechanism like no scale supergravity or conformal sequestering. Therefore, the predictive power of gauge mediation for the superpartner spectrum is generally lost. It should be said though that the situation is exactly similar to the one of the so-called anomaly-mediated supersymmetry breaking scenario [25, 26]. Finally, a larger GUT scale implies a stronger sensitivity to higher-dimensional operators suppressed by the Planck mass.

Note that one arrives at very similar conclusions in a supergravity model with a single $\mathrm{SU}(5)$ adjoint and a canonical Kähler potential [20], or in a global supersymmetric SU(5) model with two adjoints [2].

Some of these conclusions may be evaded for more complex superpotentials. ${ }^{9}$ In fact the quartic superpotential of eq. (3.7) is only a particular example. The most general

\footnotetext{
${ }^{8}$ Strictly speaking, this number is obtained from a partial 2-loop analysis, and should not be used in our 1-loop approximation. A more accurate analysis would give slightly different values for $M_{\mathrm{GUT}}$ and $m_{T}$ without changing the qualitative picture.

${ }^{9}$ Another possibility is to replace the 24 -dimensional adjoint with a 75 -dimensional representation.
} 
superpotential for $\Sigma$ involves a sum of non-renormalizable terms of the form $\Sigma^{n}, n \geq 4$, i.e. gauge invariants $\mathcal{O}_{n, k} \equiv \prod_{i}\left(\operatorname{Tr} \Sigma^{n_{i, k}}\right)^{a_{i, k}}$ with $\sum_{i} a_{i, k} n_{i, k}=n$ :

$$
W=\frac{\mu}{2} \operatorname{Tr} \Sigma^{2}+\sqrt{30} \frac{\lambda}{3} \operatorname{Tr} \Sigma^{3}+\sum_{n \geq 4} \sum_{k} \kappa_{n, k} \mathcal{O}_{n, k} .
$$

The constraints discussed in this and the previous sections can still be satisfied with a single fine-tuning between the coefficients $\kappa_{n, k}$ of the operators corresponding to a single $n$, provided that the coefficients of the other operators are small. The tuning needed is the one that makes (with the help of the small couplings $\mu, \lambda$ and $\kappa_{p, k}, p \neq n$ ) the first three derivatives of the superpotential small enough, while keeping $m_{3}$ and $m_{8}$ large enough to allow for unification below the Planck scale. The simplest and most predictive cases are $n=4$ (the case discussed so far) and $n=5$, for which there are only 2 independent operators $\mathcal{O}_{n, k}$, namely $\mathcal{O}_{4,1}=\operatorname{Tr} \Sigma^{4}, \mathcal{O}_{4,2}=\left(\operatorname{Tr} \Sigma^{2}\right)^{2}$, and $\mathcal{O}_{5,1}=\operatorname{Tr} \Sigma^{5}, \mathcal{O}_{5,2}=\left(\operatorname{Tr} \Sigma^{3}\right)\left(\operatorname{Tr} \Sigma^{2}\right)$. The triplet mass is predicted only in these two cases (with a ratio $m_{3} / m_{8}=28 / 3$ for $n=5$ ).

At this point we have all the necessary ingredients to attack another issue. The minimum obtained in the previous section is not the global minimum of the effective potential. In the limit of infinite Planck mass, one has to check whether the lifetime of this metastable vacuum can be longer than the age of the Universe. The transition rate to the $\mathrm{SU}(5)$ and supersymmetry preserving minimum at the origin can be estimated in the usual way by $e^{-S}$, where $S \approx \Delta \Phi / \Delta V \approx M_{\mathrm{GUT}}^{4} / F^{2} \approx 10^{20}$. Hence the metastability of the supersymmetry-breaking minimum is not an issue.

One could be worried that there are other supersymmetry-breaking or preserving minima, for example in the $\mathrm{SU}(4) \times \mathrm{U}(1)$ direction. This seems unlikely because the finetuning (4.5) between $\kappa_{1}$ and $\kappa_{2}$ (which is required in order to satisfy eq. (3.12) while preventing $\Sigma_{3}$ and $\Sigma_{8}$ to be light) is done in the Standard Model direction. In the $\mathrm{SU}(4) \times \mathrm{U}(1)$ direction, the relevant combination of $\kappa_{1}$ and $\kappa_{2}$ is different and gives an effective quartic coupling of order 1 , while $\mu$ and $\lambda$ remain small. This means that the $\mathrm{SU}(4) \times \mathrm{U}(1)$-invariant minimum must be very close to the origin, hence the probability for the Standard Model vacuum to decay to it is as suppressed as the probability to decay to the global SU(5)preserving minimum.

Reintroducing a finite Planck scale one would prefer to satisfy the Coleman-De Luccia bound [27] and thus avoid a possible instability of the Minkowski vacuum [16]. Although an exact calculation is beyond the scope of this paper, the vicinity of the GUT scale to the Planck scale makes this possible, as pointed out in ref. [17].

\section{$5 \quad$ Model-building aspects}

In this section, we address a few model-building issues related to the quark-lepton mass relations and to the doublet-triplet splitting problem.

\subsection{Fermion masses}

A generic problem of $\mathrm{SU}(5)$ models with a minimal Higgs sector is the equality, at the renormalizable level, between the down quark and charged lepton Yukawa couplings. As 
is well known, this leads to wrong predictions for the $m_{\mu} / m_{s}$ and $m_{e} / m_{d}$ mass ratios, and the GUT-scale relations must therefore be corrected for the first two generations (while the small discrepancy between the observed and predicted $m_{\tau} / m_{b}$ ratio may be obtained from supersymmetric threshold corrections alone).

To do this without introducing new degrees of freedom, one can just employ higherdimensional operators obtained by inserting powers of the adjoint $\Sigma$ in the standard renormalizable Yukawa coupling:

$$
W_{Y_{e}, Y_{d}}=\left(Y_{3}\right)_{i j} 10_{F}^{i} \overline{5}_{F}^{j} \overline{5}_{H}+\left(Y_{4}\right)_{i j} 10_{F}^{i}\left(\frac{\Sigma}{M_{P}} \overline{5}_{F}^{j}\right) \overline{5}_{H}+\cdots
$$

The non-renormalizable operators contribute to both the down-type Yukawa couplings and A-terms. ${ }^{10}$ For instance, eq. (5.1) gives:

$$
\begin{aligned}
\left(Y_{d}\right)_{i j} & =\left(Y_{3}\right)_{i j}+\frac{2}{\sqrt{30}} \frac{v}{M_{P}}\left(Y_{4}\right)_{i j}, & \left(Y_{e}^{T}\right)_{i j} & =\left(Y_{3}\right)_{i j}-\frac{3}{\sqrt{30}} \frac{v}{M_{P}}\left(Y_{4}\right)_{i j}, \\
\left(\delta A_{d}\right)_{i j} & =\frac{2}{\sqrt{30}} \frac{F(v)}{M_{P}}\left(Y_{4}\right)_{i j}, & \left(\delta A_{e}^{T}\right)_{i j} & =-\frac{3}{\sqrt{30}} \frac{F(v)}{M_{P}}\left(Y_{4}\right)_{i j},
\end{aligned}
$$

where the A-terms are defined by $V_{\text {soft }} \ni\left(A_{u}\right)_{i j} \tilde{Q}^{i} \tilde{U}^{c j} H_{u}+\left(A_{d}\right)_{i j} \tilde{Q}^{i} \tilde{D}^{c j} H_{d}+\left(A_{e}\right)_{i j} \tilde{L}^{i} \tilde{E}^{c j} H_{d}+$ h.c. and also receive a contribution from gauge messenger fields (see section 6 ). This typically gives large A-terms for the first and second generations, for which significant corrections to the renormalizable Yukawa couplings are required:

$$
\left(\delta A_{d, e}\right)_{i j}=\frac{F(v)}{v}\left(\delta Y_{d, e}\right)_{i j} \lesssim\left(\frac{\alpha}{4 \pi}\right)^{-1} m_{\text {soft }}\left(\delta Y_{d, e}\right)_{i j}
$$

For the second generation of leptons, one typically needs $\left(\delta Y_{e}\right)_{22} \sim\left(Y_{e}\right)_{22}$, which yields $\left|\left(\delta A_{e}\right)_{22}\right| \lesssim\left(Y_{e}\right)_{22}(\alpha / 4 \pi)^{-1} m_{\text {soft }}$, to be compared with the standard tree-level constraint $\left|A_{f}\right| \lesssim 3 y_{f} m_{\text {soft }}$ ensuring the absence of deeper charge and colour beaking (CCB) minima [28]. Although the upper bound in eq. (5.4) has little chance to be saturated, because gravitational contributions to soft terms are likely to dominate over the gauge-mediated ones (see section 6), such large A-terms are nevertheless at odds with the CCB constraints. Barring the arguments of ref. [16], however, deeper CCB minima are acceptable if the MSSM vacuum is metastable with respect to these minima. The corresponding constraints on A-terms are weaker than the ones associated with exact stability of the MSSM vacuum with respect to $\mathrm{CCB}$ minima, and moreover, they are only relevant for the A-terms associated with large Yukawa couplings [29, 30].

There is actually another type of contribution to fermion masses, which is particularly important in the case of large A-terms, coming from low-energy supersymmetric threshold corrections. These alone, according to ref. [31], could correct the wrong SU(5) mass relations. A complete analysis including these threshold corrections, with the A-terms given by eq. (5.3), on top of the contribution from eq. (5.2) is beyond the scope of this paper.

However, there is more freedom than stated above. Since the GUT scale is typically large, e.g. for the model of section $3 M_{\mathrm{GUT}} / M_{P} \gtrsim 0.05$, non-renormalizable operators

\footnotetext{
${ }^{10}$ They also contribute to the colour triplet couplings to light fermions, which may help suppress the proton decay rate.
} 
suppressed by $1 / M_{P}^{2}$ may be relevant even to the determination of the second generation Yukawa couplings. Schematically, one has:

$$
\begin{aligned}
Y & =Y_{3}+x\left(c_{4} Y_{4}+c_{5} x Y_{5}\right), \\
\delta A & =\frac{F(v)}{v} x\left(c_{4} Y_{4}+2 c_{5} x Y_{5}\right),
\end{aligned}
$$

where $x \equiv M_{\mathrm{GUT}} / \sqrt{30} M_{P}$ and $c_{4}, c_{5}$ are coefficients of order 1 . Due to the factor of 2 in eq. (5.6), the correlation between the A-terms and the corrections to the renormalizable Yukawa couplings is spoiled. This makes it possible to ensure the stability of the MSSM vacuum with respect to CCB minima, as required by the arguments of refs. [16, 17].

\subsection{Doublet-triplet splitting}

Since the adjoint $\Sigma$ acquires a VEV along both its lowest and F-term components, two fine-tunings are a priori necessary: one to split the $\mu$ term from the triplet mass, another one to split the $B \mu$ term from the scalar triplet-antitriplet mixing parameter, which is of order $F$. To perform this double splitting, one needs to go beyond the renormalizable level:

$$
W=\bar{H}\left(M_{H}+\eta \Sigma+\frac{\beta_{1}}{M} \Sigma^{2}+\frac{\beta_{2}}{M} \operatorname{Tr}\left(\Sigma^{2}\right)\right) H
$$

where $H \equiv 5_{H}$ and $\bar{H} \equiv \overline{5}_{H}$. The two fine-tunings are:

$$
\begin{aligned}
& M_{H}-\sqrt{\frac{3}{10}} \eta v+\left(\frac{3}{10} \beta_{1}+\beta_{2}\right) \frac{v^{2}}{M}=0, \\
&-\sqrt{\frac{3}{10}} \eta+2\left(\frac{3}{10} \beta_{1}+\beta_{2}\right) \frac{v}{M}=0,
\end{aligned}
$$

and as mentioned above require that at least one of the two parameters $\beta_{1}$ and $\beta_{2}$ be non vanishing. The triplet mass is thus given by:

$$
\begin{aligned}
m_{T} & =M_{H}+\frac{2}{\sqrt{30}} \eta v+\left(\frac{4}{30} \beta_{1}+\beta_{2}\right) \frac{v^{2}}{M} \\
& =\left(\frac{5}{6} \beta_{1}+\frac{10}{3} \beta_{2}\right) \frac{v^{2}}{M} .
\end{aligned}
$$

The doublet-triplet splitting can be performed in two ways:

1. The first one sticks to the principle of minimal fine-tuning. If one chooses $M_{H}=\eta=$ 0 , a single fine-tuning is needed in order to satisfy both eqs. (5.8) and (5.9), namely $3 \beta_{1} / 10=-\beta_{2}$. The triplet mass then reads $m_{T}=-\left(\beta_{1} / 6\right)\left(M_{\mathrm{GUT}}^{2} / M_{P}\right)$. To achieve $m_{T}=10^{17} \mathrm{GeV}$ as in the model of section 3 , one needs $M_{\mathrm{GUT}} \simeq 10^{18} \mathrm{GeV} / \sqrt{\left|\beta_{1}\right|}$, so that the perturbative expansion in powers of $M_{\mathrm{GUT}} / M_{P}$ can no longer be trusted (either because $M_{\mathrm{GUT}} / M_{P}$ is not a small parameter, or because $\beta_{1}$ is non-perturbative). Perturbativity thus forces one to consider a lower value of the triplet mass, however still consistent with the RGE analysis of ref. [24]. One then has to invoke another way of suppressing proton decay (see e.g. ref. [23]). 
2. The second possibility is to choose $\beta_{1}$ and $\beta_{2}$ so as to maximize the triplet mass. This is done at the expense of a second fine-tuning, the combination $3 \beta_{1} / 10+\beta_{2}$ being fixed in eqs. (5.8) and (5.9). For instance, $m_{T}=10^{17} \mathrm{GeV}$ can be achieved with $\beta_{1}=\beta_{2}=1$ and $M_{\mathrm{GUT}}=2.4 \times 10^{17} \mathrm{GeV}$.

An alternative possibility is to use the missing partner mechanism [32, 33]. This requires replacing the adjoint $\Sigma$ with a $75_{H}$ and adding a pair of $50_{H} \oplus \overline{50}_{H}$. In this case the doublet-triplet splitting is realized for both $\mu$ and $B \mu$ without fine-tuning. The spontaneous breaking of supersymmetry and of the $\mathrm{SU}(5)$ gauge symmetry by the $75_{H}$ proceeds as described in section 3, but with a different value of $c$. The wrong SU(5) mass relations can be corrected by non-renormalizable operators involving powers of the $75_{H}$. The main differences with the adjoint case are the spectrum of intermediate states left over from the $75_{H}$, the running of gauge couplings (section 4 ), and the predictions for the MSSM soft terms (section 6).

\section{The superpartner spectrum}

In this section, we discuss the gauge-mediated contributions to the MSSM soft terms in the SU(5) model described in section 3. Before doing so, let us stress that the supergravity contributions will generally dominate due to the large messenger scale, $M_{\mathrm{GUT}} \sim 10^{17} \mathrm{GeV}$. Indeed, the typical size of a gravity-mediated soft term is $m_{3 / 2}=F / \sqrt{3} M_{P}$, which is about one order of magnitude larger than $(\alpha / 4 \pi) F / M_{\mathrm{GUT}}$. Nevertheless, some scenarios like the sequestering mechanism discussed in ref. [34] lead to a suppression of the supergravity contributions, such that the MSSM soft terms predominantly arise from messenger loops. In this section we shall assume that such a mechanism is at work. The gaugino and scalar soft masses then receive two types of contributions: (i) gauge messenger contributions arising from loops of $X$ and $Y$ gauge fields, and (ii) chiral messenger contributions from (a) the weak triplet and colour octet components of $\Sigma$, and (b) the colour Higgs triplet and antitriplet:

$$
\begin{aligned}
& m_{\chi}^{2}\left(M_{\mathrm{GUT}}\right)=\Delta_{\mathrm{GM}} m_{\chi}^{2}+\Delta_{\left(\Sigma_{3}, \Sigma_{8}\right)} m_{\chi}^{2}+\Delta_{(T, \bar{T})} m_{\chi}^{2} \\
& M_{a}\left(M_{\mathrm{GUT}}\right)=\Delta_{\mathrm{GM}} M_{a}+\Delta_{\left(\Sigma_{3}, \Sigma_{8}\right)} M_{a}+\Delta_{(T, \bar{T})} M_{a} .
\end{aligned}
$$

Each contribution can be computed using the wave function renormalization technique [7, 35]. For gauge messengers we find, in agreement with the literature:

$$
\begin{aligned}
& \Delta_{\mathrm{GM}} m_{\chi}^{2}\left(M_{\mathrm{GUT}}\right)=\left(\frac{\alpha_{\mathrm{GUT}}}{4 \pi}\right)^{2} 2\left[b_{\mathrm{SU}(5)} \Delta c^{\chi}-\sum_{a} \Delta b_{a} c_{a}^{\chi}\right]\left|\frac{F}{M_{\mathrm{GUT}}}\right|^{2}, \\
& \Delta_{\mathrm{GM}} M_{a}\left(M_{\mathrm{GUT}}\right)=-\frac{\alpha_{\mathrm{GUT}}}{4 \pi} \Delta b_{a} \frac{F}{M_{\mathrm{GUT}}},
\end{aligned}
$$

where $\Delta b_{a}=2\left(C[\mathrm{SU}(5)]-C\left[G_{a}\right]\right)$ is the contribution of the massive $X$ and $Y$ vector multiplets to the beta function coefficient of the gauge group $G_{a}\left(G_{a}=\mathrm{SU}(3)_{c}, \mathrm{SU}(2)_{L}, \mathrm{U}(1)_{Y}\right.$ for $a=1,2,3$, respectively), $\Delta c^{\chi}=c_{\mathrm{SU}(5)}^{\chi}-\sum_{a} c_{a}^{\chi}$ is the difference between the second Casimir coefficients of the $\mathrm{SU}(5)$ and $\mathrm{SU}(3)_{C} \times \mathrm{SU}(2)_{L} \times \mathrm{U}(1)_{Y}$ representations to which 
$\chi$ belongs, the normalization of the hypercharge generator is the $\mathrm{SU}(5)$ one and we have identified $v=M_{\mathrm{GUT}}$. For $\Sigma_{3}, \Sigma_{8}$ and $(T, \bar{T})$, one obtains the standard chiral messenger formula, with ${ }^{11} F /\left.M\right|_{\Sigma_{3}}=F /\left.M\right|_{\Sigma_{8}}=2 F / M_{\mathrm{GUT}}$. Note that the triplet contribution depends on how the doublet-triplet splitting is realized; here we assume that this is done with a single fine-tuning in the way described in section 5.2 , in which case one also has $F /\left.M\right|_{(T, \bar{T})}=2 F / M_{\mathrm{GUT}}$.

The resulting gaugino and scalar soft masses at the messenger scale are given in the table below, together with the values of each individual contribution, in units of

$$
m_{\mathrm{GM}}=\frac{\alpha_{\mathrm{GUT}}}{4 \pi} \frac{F}{M_{\mathrm{GUT}}} .
$$

\begin{tabular}{|c|c|c|c|c|c|c|}
\hline$m_{\chi}^{2} / m_{\mathrm{GM}}^{2}$ & $Q$ & $U^{c}$ & $E^{c}$ & $L$ & $D^{c}$ & $H_{u}, H_{d}$ \\
\hline$\Delta_{\mathrm{GM}}$ & -11 & -4 & 6 & -3 & -6 & -3 \\
\hline$\Delta_{\left(\Sigma_{3}, \Sigma_{8}\right)}$ & 44 & 32 & 0 & 12 & 32 & 12 \\
\hline$\Delta_{(T, \bar{T})}$ & $\frac{804}{75}$ & $\frac{864}{75}$ & $\frac{48}{25}$ & $\frac{12}{25}$ & $\frac{816}{75}$ & $\frac{12}{25}$ \\
\hline total & $\frac{1093}{25}$ & $\frac{988}{25}$ & $\frac{198}{25}$ & $\frac{237}{25}$ & $\frac{922}{25}$ & $\frac{237}{25}$ \\
\hline
\end{tabular}

\begin{tabular}{|c|c|c|c|}
\hline$M_{a} / m_{\mathrm{GM}}$ & $M_{3}$ & $M_{2}$ & $M_{1}$ \\
\hline$\Delta_{\mathrm{GM}}$ & -4 & -6 & -10 \\
\hline$\Delta_{\left(\Sigma_{3}, \Sigma_{8}\right)}$ & 6 & 4 & 0 \\
\hline$\Delta_{(T, \bar{T})}$ & 2 & 0 & $\frac{4}{5}$ \\
\hline total & 4 & -2 & $-\frac{46}{5}$ \\
\hline
\end{tabular}

As for A-terms, they only receive contributions from gauge messengers:

$$
A_{\chi}\left(M_{\mathrm{GUT}}\right)=\frac{\alpha_{\mathrm{GUT}}}{4 \pi} 2 \Delta c^{\chi} \frac{F}{M_{\mathrm{GUT}}},
$$

with $A_{u}=A_{Q}+A_{U^{c}}+A_{H_{u}}$, etc, yielding:

$$
A_{u}\left(M_{\mathrm{GUT}}\right)=10 m_{\mathrm{GM}}, A_{d}\left(M_{\mathrm{GUT}}\right)=8 m_{\mathrm{GM}}, A_{e}\left(M_{\mathrm{GUT}}\right)=12 m_{\mathrm{GM}} .
$$

We have assumed the minimal field content, with only the adjoint $\Sigma$ and a pair of Higgs fields in the $5 \oplus \overline{5}$ representation (giving $b_{\mathrm{SU}(5)}=3$ ), and neglected the running of the gauge couplings between the various messenger scales $M_{\mathrm{GUT}}, m_{3}, m_{8}$ and $m_{T}$.

A few comments are in order. First, the negative gauge messenger contributions to scalar squared masses are smaller than the positive contributions from chiral messengers, so that there is no tachyonic mass at the messenger scale. ${ }^{12}$ Second, the soft terms are rather large in units of $m_{\mathrm{GM}}$, implying that even a moderate suppression of supergravity corrections may be enough for gauge-mediated contributions to dominate.

Let us briefly comment on the physical superpartner spectrum obtained by running the above boundary conditions at the messenger scale down to the electroweak scale. If we require squarks to be lighter than $2 \mathrm{TeV}$ or so, the lightest Higgs boson mass will be typically below $120 \mathrm{GeV}$, despite the fact that a rather large $A_{t}$ is generated at the messenger scale. A Higgs mass around the value indicated by the ATLAS [36] and CMS [37]

\footnotetext{
${ }^{11}$ It should be stressed that $F /\left.M\right|_{\Sigma_{3}}$ and $F /\left.M\right|_{\Sigma_{8}}$ are model dependent. The factor of 2 relative to $F / M_{\mathrm{GUT}}$ is specific to the quartic superpotential of eq. (3.7).

${ }^{12}$ This is different from ref. [34], in which chiral messenger contributions are omitted, and the renormalization group running is crucial to make all sfermion squared masses positive at the electroweak scale.
} 


\begin{tabular}{|c|c|c|c|c|c|c|c|c|c|c|}
\hline $\mathrm{h}$ & $\mathrm{A}$ & $H^{0}$ & $H^{ \pm}$ & $\tilde{\chi}_{1}^{ \pm}$ & $\tilde{\chi}_{2}^{ \pm}$ & $\tilde{\chi}_{1}^{0}$ & $\tilde{\chi}_{2}^{0}$ & $\tilde{\chi}_{3}^{0}$ & $\tilde{\chi}_{4}^{0}$ & $\tilde{g}$ \\
\hline 124.75 & 6267.8 & 6267.8 & 6268.2 & 1750 & 6002 & 1750 & 3743 & 6001 & 6002 & 8296 \\
\hline
\end{tabular}

\begin{tabular}{|c|c|c|c|c|c|c|c|}
\hline$\tilde{t}_{1}$ & $\tilde{t}_{2}$ & $\tilde{u}_{1}, \tilde{c}_{1}$ & $\tilde{u}_{2}, \tilde{c}_{2}$ & $\tilde{b}_{1}$ & $\tilde{b}_{2}$ & $\tilde{d}_{1}, \tilde{s}_{1}$ & $\tilde{d}_{2}, \tilde{s}_{2}$ \\
\hline 7421 & 8350 & 9275 & 9295 & 8330 & 8726 & 8949 & 9296 \\
\hline
\end{tabular}

\begin{tabular}{|c|c|c|c|c|c|}
\hline$\tilde{\tau}_{1}$ & $\tilde{\tau}_{2}$ & $\tilde{e}_{1}, \tilde{\mu}_{1}$ & $\tilde{e}_{2}, \tilde{\mu}_{2}$ & $\tilde{\nu}_{\tau}$ & $\tilde{\nu}_{e}, \tilde{\nu}_{\mu}$ \\
\hline 3050 & 3361 & 3557 & 4236 & 3053 & 3556 \\
\hline
\end{tabular}

Table 1. Superpartner masses (in GeV) predicted by the SU(5) model of section 3, assuming suppressed supergravity contributions and doublet-triplet splitting through a single fine-tuning. The input parameters are $M_{\mathrm{GUT}}=10^{17} \mathrm{GeV}, b_{\mathrm{SU}(5)}=3, m_{\mathrm{GM}}=960 \mathrm{GeV}, \tan \beta=25$ and $\operatorname{sign}(\mu)=+$.

experiments requires significantly heavier squarks and sleptons (unless other contributions to the top squark A-term are invoked, e.g. from a non-renormalizable superpotential term $\left.10_{F}^{3} 10_{F}^{3} \Sigma 5_{H} / M_{P}\right)$. As an example, we display in table 1 the spectrum corresponding to the choice $m_{\mathrm{GM}}=960 \mathrm{GeV}, \tan \beta=25$ and $\mu>0$ (with $\mu$ and $B \mu$ determined from radiative electroweak symmetry breaking) obtained with the help of the code SuSpect [38]. The parameters $m_{3}=4 m_{8}$ and $m_{T}$ were chosen in such a way that gauge coupling unification occurs at $M_{\mathrm{GUT}}=10^{17} \mathrm{GeV}$, but the effect of the corresponding thresholds on the running of the soft terms has been neglected. A noticeable feature of this spectrum is that the LSP is the wino (the gravitino mass being $6.4 \mathrm{TeV}$ ), with a mass in the right ballpark to account for the observed dark matter relic density. Unfortunately, this spectrum cannot be tested at the LHC.

\section{Conclusions}

One of the main obstructions in constructing predictive supersymmetric Grand Unified Theories is our ignorance of the way supersymmetry is broken. This leaves proton decay rates as well as the fermion mass fitting undetermined, since these observables depend on the way supersymmetry is broken and mediated to the MSSM sector. A crucial step towards predictive supersymmetric unification is therefore to implement supersymmetry breaking and its mediation in realistic GUTs.

In this paper, we showed that it is possible to break simultaneously supersymmetry and the gauge symmetry with a single field, in spite of the absence of a tree-level flat direction. The local supersymmetry-breaking minimum is induced radiatively in the effective potential, far away from the gauge- and supersymmetry-preserving global minimum, in a region where the tree-level potential is almost flat. The condition for this mechanism to work is a fine-tuned correlation between the superpotential parameters. Supersymmetry breaking is then transmitted to the MSSM through both gauge and chiral messenger loops, thus avoiding the suppression of gaugino masses characteristic of direct gauge mediation 
models [13]. The use of a single field ensures that no dangerous tachyonic scalar masses are generated at the one-loop level.

We studied the implementation of this mechanism in an SU(5) model with a single adjoint representation. A generic prediction is that intermediate states are unavoidable, which raises the GUT scale and allows for a larger Higgs triplet mass, as preferred by the experimental lower bound on the proton lifetime. In the particular case of a quartic superpotential, the Higgs triplet mass is even predicted, but a strong fine-tuning among the couplings of the quartic terms is needed to achieve unification below the Planck scale. An unfortunate consequence of the increase of the GUT scale is that supergravity contributions to the MSSM soft terms will generally dominate over the gauge-mediated ones, unless one relies on a mechanism like no-scale supergravity or conformal sequestering. Only in this case can one obtain definite predictions for the superpartner spectrum. These were presented in section 6. We also discussed model-building aspects related to the quark-lepton mass relations and to the doublet-triplet splitting, which present significant differences with the case of separate supersymmetry and gauge symmetry breaking sectors.

Similar predictions to the ones found in this paper are obtained in a supergravity model with a single SU(5) adjoint, in which supersymmetry and the gauge symmetry are broken at the tree level [20]. It would be interesting to investigate how the mechanism that we have proposed would be affected by local supersymmetry. Also, it remains to be studied how it can be embedded in a higher-rank Grand Unified Theory, possibly avoiding the strong tuning among superpotential parameters present in the minimal SU(5) case.

\section{Acknowledgments}

We thank K.S. Babu for discussions. This work has been supported in part by the Slovenian Research Agency, by the Agence Nationale de la Recherche under Grant ANR 2010 BLANC 0413 01, by the European Commission under Contract PITN-GA-2009-237920, and by the French-Slovenian programme Proteus under Contracts BI-FR/10-11-PROTEUS-14 and 22773RG. BB and SL acknowledge partial financial support from the NSF under Grant No. 1066293 and the hospitality of the Aspen Center for Physics. SL thanks the Galileo Galilei Institute for Theoretical Physics for hospitality and the INFN for partial support.

Open Access. This article is distributed under the terms of the Creative Commons Attribution License which permits any use, distribution and reproduction in any medium, provided the original author(s) and source are credited.

\section{References}

[1] E. Witten, Mass hierarchies in supersymmetric theories, Phys. Lett. B 105 (1981) 267 [INSPIRE].

[2] B. Bajc and A. Melfo, Metastable gauged O'Raifeartaigh, JHEP 04 (2008) 062 [arXiv: 0801.4349] [INSPIRE].

[3] M. Dine and W. Fischler, A supersymmetric GUT, Nucl. Phys. B 204 (1982) 346 [INSPIRE]. 
[4] S. Dimopoulos and S. Raby, Geometric hierarchy, Nucl. Phys. B 219 (1983) 479 [inSPIRE].

[5] T. Banks and V. Kaplunovsky, Nosonomy of an upside down hierarchy model. 1, Nucl. Phys. B 211 (1983) 529 [INSPIRE].

[6] J. Derendinger and C.A. Savoy, Gaugino masses and a new mechanism for proton decay in supersymmetric theories, Phys. Lett. B 118 (1982) 347 [INSPIRE].

[7] V. Kaplunovsky, Nosonomy of an upside down hierarchy model. 2, Nucl. Phys. B 233 (1984) 336 [INSPIRE].

[8] H. Murayama, A model of direct gauge mediation, Phys. Rev. Lett. 79 (1997) 18 [hep-ph/9705271] [INSPIRE].

[9] S. Dimopoulos, G. Dvali, R. Rattazzi and G. Giudice, Dynamical soft terms with unbroken supersymmetry, Nucl. Phys. B 510 (1998) 12 [hep-ph/9705307] [INSPIRE].

[10] M.A. Luty, Simple gauge mediated models with local dynamical supersymmetry breaking, Phys. Lett. B 414 (1997) 71 [hep-ph/9706554] [InSPIRE].

[11] K. Agashe, GUT and SUSY breaking by the same field, Phys. Lett. B 444 (1998) 61 [hep-ph/9809421] [INSPIRE].

[12] K. Agashe, Improved GUT and SUSY breaking by the same field, Nucl. Phys. B 588 (2000) 39 [hep-ph/0003236] [INSPIRE].

[13] Z. Komargodski and D. Shih, Notes on SUSY and R-symmetry breaking in Wess-Zumino models, JHEP 04 (2009) 093 [arXiv:0902.0030] [INSPIRE].

[14] A. Giveon, A. Katz and Z. Komargodski, Uplifted metastable vacua and gauge mediation in SQCD, JHEP 07 (2009) 099 [arXiv: 0905.3387] [INSPIRE].

[15] E. Dudas, S. Lavignac and J. Parmentier, On messengers and metastability in gauge mediation, Phys. Lett. B 698 (2011) 162 [arXiv:1011.4001] [INSPIRE].

[16] G. Dvali, Safety of Minkowski vacuum, arXiv:1107.0956 [INSPIRE].

[17] B. Bajc, G. Dvali and G. Senjanović, Problems with false vacua in supersymmetric theories, arXiv:1108.5195 [INSPIRE].

[18] K. Intriligator and M. Sudano, General gauge mediation with gauge messengers, JHEP 06 (2010) 047 [arXiv: 1001.5443] [INSPIRE].

[19] S.R. Coleman and E.J. Weinberg, Radiative corrections as the origin of spontaneous symmetry breaking, Phys. Rev. D 7 (1973) 1888 [INSPIRE].

[20] B. Bajc and G. Senjanović, Proton decay, supersymmetry breaking and its mediation, Phys. Lett. B 648 (2007) 365 [hep-ph/0611308] [INSPIRE].

[21] C. Bachas, C. Fabre and T. Yanagida, Natural gauge coupling unification at the string scale, Phys. Lett. B 370 (1996) 49 [hep-th/9510094] [INSPIRE].

[22] J. Chkareuli and I. Gogoladze, Unification picture in minimal supersymmetric SU(5) model with string remnants, Phys. Rev. D 58 (1998) 055011 [hep-ph/9803335] [INSPIRE].

[23] B. Bajc, P. Fileviez Perez and G. Senjanović, Minimal supersymmetric SU(5) theory and proton decay: where do we stand?, hep-ph/0210374 [INSPIRE].

[24] H. Murayama and A. Pierce, Not even decoupling can save minimal supersymmetric SU(5), Phys. Rev. D 65 (2002) 055009 [hep-ph/0108104] [INSPIRE]. 
[25] L. Randall and R. Sundrum, Out of this world supersymmetry breaking, Nucl. Phys. B 557 (1999) 79 [hep-th/9810155] [INSPIRE].

[26] G.F. Giudice, M.A. Luty, H. Murayama and R. Rattazzi, Gaugino mass without singlets, JHEP 12 (1998) 027 [hep-ph/9810442] [INSPIRE].

[27] S.R. Coleman and F. De Luccia, Gravitational effects on and of vacuum decay, Phys. Rev. D 21 (1980) 3305 [INSPIRE].

[28] J. Frere, D. Jones and S. Raby, Fermion masses and induction of the weak scale by supergravity, Nucl. Phys. B 222 (1983) 11 [INSPIRE].

[29] M. Claudson, L.J. Hall and I. Hinchliffe, Low-energy supergravity: false vacua and vacuous predictions, Nucl. Phys. B 228 (1983) 501 [INSPIRE].

[30] A. Kusenko, P. Langacker and G. Segre, Phase transitions and vacuum tunneling into charge and color breaking minima in the MSSM, Phys. Rev. D 54 (1996) 5824 [hep-ph/9602414] [INSPIRE].

[31] T. Enkhbat, SU(5) unification for Yukawas through SUSY threshold effects, arXiv:0909.5597 [INSPIRE].

[32] A. Masiero, D.V. Nanopoulos, K. Tamvakis and T. Yanagida, Naturally massless Higgs doublets in supersymmetric SU(5), Phys. Lett. B 115 (1982) 380 [InSPIRE].

[33] B. Grinstein, A supersymmetric $\mathrm{SU}(5)$ gauge theory with no gauge hierarchy problem, Nucl. Phys. B 206 (1982) 387 [INSPIRE].

[34] R. Dermisek, H.D. Kim and I.-W. Kim, Mediation of supersymmetry breaking in gauge messenger models, JHEP 10 (2006) 001 [hep-ph/0607169] [INSPIRE].

[35] G. Giudice and R. Rattazzi, Extracting supersymmetry breaking effects from wave function renormalization, Nucl. Phys. B 511 (1998) 25 [hep-ph/9706540] [InSPIRE].

[36] ATLAS collaboration, G. Aad et al., Combined search for the standard model Higgs boson using up to $4.9 \mathrm{fb}^{-1}$ of $\mathrm{pp}$ collision data at $\sqrt{\mathrm{s}}=7 \mathrm{TeV}$ with the ATLAS detector at the LHC, Phys. Lett. B $\mathbf{7 1 0 ~ ( 2 0 1 2 )} 49$ [arXiv:1202.1408] [INSPIRE].

[37] CMS collaboration, S. Chatrchyan et al., Combined results of searches for the standard model Higgs boson in pp collisions at $\sqrt{s}=7$ TeV, Phys. Lett. B 710 (2012) 26 [arXiv: 1202.1488] [INSPIRE].

[38] A. Djouadi, J.-L. Kneur and G. Moultaka, SuSpect: a Fortran code for the supersymmetric and Higgs particle spectrum in the MSSM, Comput. Phys. Commun. 176 (2007) 426 [hep-ph/0211331] [INSPIRE]. 